\title{
Transition from blastomere to trophectoderm biopsy: comparing two preimplantation genetic testing for aneuploidies strategies
}

\author{
Lluc Coll1 ${ }^{1}$, Mònica Parriego ${ }^{2}$, Montserrat Boada², Marta Devesa², Gemma Arroyo², \\ Ignacio Rodríguez², Bonaventura Coroleu ${ }^{2}$, Francesca Vidal ${ }^{3}$ and Anna Veiga ${ }^{2,4}$ \\ Reproductive Medicine Service, Department of Obstetrics, Gynaecology and Reproduction, Hospital Universitari Dexeus - \\ Dexeus Women's Health, Barcelona; Unitat de Biologia Cellular, Facultat de Biociències, Universitat Autònoma de Barcelona, \\ Bellaterra; and Stem Cell Bank, Centre of Regenerative Medicine in Barcelona, Barcelona, Spain
}

Date submitted: 31.08.2017. Date revised: 11.01.2018. Date accepted: 15.03.2018

\section{Summary}

Shortly after the implementation of comprehensive chromosome screening (CCS) techniques for preimplantation genetic testing for aneuploidies (PGT-A), the discussion about the transition from day 3 to blastocyst stage biopsy was initiated. Trophectoderm biopsy with CCS is meant to overcome the limitations of cleavage-stage biopsy and single-cell analysis. The aim of this study was to assess the results obtained in our PGT-A programme after the implementation of this new strategy. Comparisons between the results obtained in 179 PGT-A cycles with day 3 biopsy $(\mathrm{D}+3)$ and fresh embryo transfer, and 204 cycles with trophectoderm biopsy and deferred (frozen-thawed) embryo transfer were established. Fewer embryos were biopsied and a higher euploidy rate was observed in the trophectoderm biopsy group. No differences in implantation $(50.3 \%$ vs. $61.4 \%)$ and clinical pregnancy rate per transfer $(56.1 \%$ vs. $65.3 \%$ ) were found. Although the mean number of euploid embryos per cycle did not differ between groups $(1.5 \pm 1.7$ vs. $1.7 \pm 1.8)$, the final number of euploid blastocysts available for transfer per cycle was significantly higher in the trophectoderm biopsy group (1.1 $\pm 1.3 \mathrm{vs} .1 .7 \pm 1.8)$. This factor led to an increased cumulative live birth rate in this last group (34.1\% vs. $44.6 \%$ ). Although both strategies can offer good results, trophectoderm biopsy offers a more robust diagnosis and the intervention is less harmful for the embryos so more euploid blastocysts are finally available for transfer and/or vitrification.

Keywords: Aneuploidy, Cleavage stage, Blastocyst, Biopsy, In vitro fertilization, Live birth rate, Preimplantation genetic testing

\section{Introduction}

In the past decade preimplantation genetic testing for aneuploidies (PGT-A) has undergone two significant

\footnotetext{
${ }^{1}$ All correspondence to: Lluc Coll. Gran Via Carles III, 71-75. 08028 Barcelona, Spain. Tel: +34932274700 ext.22176. E-mail: 1lucol@dexeus.com

${ }^{2}$ Reproductive Medicine Service, Department of Obstetrics, Gynaecology and Reproduction, Hospital Universitari Dexeus - Dexeus Women's Health. Gran Via Carles III, 71-75, 08028 Barcelona, Spain.

${ }^{3}$ Unitat de Biologia Cellular, Facultat de Biociències, Universitat Autònoma de Barcelona, 08193 Bellaterra, Spain.

${ }^{4}$ Stem Cell Bank. Centre of Regenerative Medicine in Barcelona. Gran Via de l'Hospitalet 199, 08908, Barcelona, Spain.
}

changes: one regarding the technology used for the analysis and the other concerning timing of biopsy.

As the early 1990s, when PGT-A was first described (Munné et al., 1993), the technique used for analysis was fluorescence in situ hybridization (FISH). Its main limitation was the impossibility to screen all chromosomes. This fact, along with randomized clinical trials pointing to PGT-A as detrimental (Mastenbroek et al., 2007, 2011) opened the debate. The emergence of comprehensive chromosome screening (CCS) techniques optimized for single-cell analysis and clinical use (Wells et al., 2008; Treff \& Scott, 2012; Handyside, 2013) set a new era for PGT-A.

Shortly after the transition from FISH to CCS, the transition from blastomere to trophectoderm biopsy arose. As early as 1990, the usefulness of trophectoderm cells for preimplantation genetic testing (PGT) in 
overcoming the limitations of single-cell analysis was suggested (Dokras et al., 1990). However, not until 2004 was trophectoderm biopsy strategy implemented and routinely performed at an in vitro fertilization (IVF) centre (de Boer et al., 2004). Improvements in culture medium and conditions, the use of laser technology for embryo biopsy (Veiga et al., 1997; Boada et al., 1998), and the rise of vitrification as a new cryopreservation tool (Kuwayama et al., 2005) made trophectoderm biopsy a realistic and efficient option for clinical practice. More recently, time-lapse monitoring has also contributed to biopsy at the blastocyst stage in terms of undisturbed culture conditions and assessing the best moment to perform the biopsy (Capalbo et al., 2016).

As presented above, the limitations of single-cell analysis can be overcome by trophectoderm biopsy. In this latter approach, in which 3-8 cells are biopsied, diagnosis is more robust and its failure more unlikely (Forman et al., 2012). Moreover, trophectoderm biopsy has also been reported to be less harmful to the embryo (Scott et al., 2013). In terms of embryo mosaicism, blastocyst biopsy allows its detection at certain levels (from 20\%) depending on the CCS technique used (Mamas et al., 2012; PGDIS, 2016).

Fresh euploid blastocyst transfer strategy or frozenthawed euploid blastocyst transfer policies can be followed (Coates et al., 2017).

Published results on clinical outcomes obtained with PGT-A with trophectoderm biopsy are promising (Dahdouh et al., 2015) and are encouraging many groups to change their $\mathrm{D}+3$ biopsy programmes to blastocyst biopsy.

There are very few publications that compare $\mathrm{D}+3$ and trophectoderm biopsy, analysing the changes experienced in a PGT-A programme by changing the biopsy timing (Harton et al., 2013; Adler et al., 2014).

The aim of this study was to compare the results obtained in our PGT-A programme by two different approaches: single-cell D+3 biopsy and fresh embryo transfer on day 5 and trophectoderm biopsy and deferred (frozen-thawed) embryo transfer.

\section{Materials and Methods}

In total, 383 PGT-A cycles with their own gametes between January 2014 and February 2016 were included and analysed retrospectively.

Here, 172 patients undergoing 179 cycles were included in the D+3 biopsy group and 193 patients undergoing 204 cycles were included in the trophectoderm biopsy group. Patients had normal karyotypes and underwent a PGT-A cycle for one of the following indications: advanced maternal age ( $>37$ years), severe male factor with altered sperm FISH analysis, repeated implantation failure ( $\geq 3$ transfers without implantation or $\geq 5$ optimal quality transferred embryos failing to implant), recurrent miscarriages $(\geq 3)$, and previous pregnancies affected of chromosomopathies.

\section{Ovarian stimulation and oocyte retrieval}

Patients underwent pharmacological treatment of ovulation induction by GnRH agonists or antagonists. Both ovarian stimulation protocols and monitoring of follicular growth and hormonal oestradiol blood levels followed the standards applied routinely in our centre as previously described (Barri et al., 2002). Oocyte retrieval was performed under transvaginal ultrasound guidance $36 \mathrm{~h}$ after hCG administration.

\section{Gamete and embryo manipulation, PGT-A procedures and embryo transfer}

Gametes and embryos were cultured in LifeGlobal ${ }^{\circledR}$ medium following the manufacturer's instructions. Mature oocytes were inseminated by intracytoplasmic sperm injection (ICSI) $40 \mathrm{~h}$ after hCG administration and then cultured in a time-lapse incubator (Embryoscope $\left.{ }^{\circledR}\right)$.

All PGT-A cycles were performed during a relatively short interval of time (January 2014 to February 2016). Three periods can be identified. During the first period, PGT-A was routinely performed with single-cell biopsy on $\mathrm{D}+3$ and fresh euploid embryo transfer on day 5. After proper training of the embryologists, the optimization of laboratory organization, and the successful performance of some pilot cases, the decision to embrace a PGT-A strategy with trophectoderm biopsy and deferred embryo transfer was made. During the second period, both strategies coexisted. Finally, there was a third period when the transition was complete and all the cases were performed with blastocyst biopsy and deferred euploid embryo transfer.

\section{Cleavage-stage biopsy (Group 1)}

Embryos presenting $\geq 5$ cells and $<30 \%$ fragmentation at $66 \pm 2 \mathrm{~h}$ post-ICSI were considered suitable for biopsy. Embryos with $<5$ cells and/or $>30 \%$ fragmentation were discarded. Zona opening was performed by laser thermolysis (Boada et al., 1998) and the biopsy was carried out by aspiration of a single blastomere. Whole genome amplification, processing and analysis were performed using the kits, software, and protocols for array comparative genomic hybridization (aCGH) analysis provided by the manufacturer (SurePlex®) DNA Amplification System, 24Sure ${ }^{\circledR}$ Microarray Pack, Bluefuse ${ }^{\circledR}$, Illumina $\left.{ }^{\circledR}\right)$. One or two euploid embryos were replaced under ultrasound control (Coroleu et al., 2006) on day 5. Remaining euploid blastocysts were vitrified using the kits and protocols provided by the manufacturer (Kitazato $\left.{ }^{\circledR}\right)$. Luteal phase support was 
achieved with vaginal progesterone ( $200 \mathrm{mg}$ every $8 \mathrm{~h}$ ) until the determination of beta-hCG in plasma ( 15 days after oocyte retrieval). For positive results, the support treatment continued until ultrasound detection of fetal heartbeat on the sixth week of pregnancy.

\section{Trophectoderm biopsy (Group 2)}

Zona opening was performed on D+3 embryos with the same criteria and methodology described for Group 1. These embryos were left in culture and those developing to the blastocyst stage between day 5 and day 7 were biopsied using laser technology as previously described (Veiga et al., 1997). Only blastocysts with a well defined inner cell mass and with a hatching trophectoderm with multiple cells were considered eligible for biopsy. Three to eight trophectoderm cells were biopsied. Blastocysts were vitrified immediately after the biopsy (Kitazato ${ }^{\circledR}$ ). Analysis by aCGH was performed as in Group 1. One or two euploid blastocysts were replaced in a deferred cycle. Endometrial preparation and laboratory procedures for deferred embryo transfer were performed as previously reported (Parriego et al., 2007). Embryo transfer and follow up did not differ from the methodology described for Group 1.

\section{Statistical analysis}

The Wilcoxon Mann-Whitney non-parametric test was used to compare continuous variables among the groups. Categorical variables were compared using the chi-squared test or by comparison of their confidence interval at $95 \%$ (95\% CI).

All statistical analyses were performed using SAS software (Copyright (C) 2002-2012; SAS Institute Inc. SAS and all other SAS Institute Inc. product or service names are registered trademarks or trademarks of SAS Institute Inc., Cary, NC, USA). $P$-values $<0.05$ were considered to indicate statistical significance as well as non-overlapping 95\% CI.

\section{Results}

In total, 383 PGT-A cycles were included: 179 cycles with cleavage-stage biopsy (Group 1) and 204 cycles with trophectoderm biopsy (Group 2). The indications for PGT-A in both groups were comparable: $48.6 \%$ vs. $56.9 \%$ for advanced maternal age, $17.3 \%$ vs. $17.1 \%$ for male factor, $16.2 \%$ vs. $14.7 \%$ for repeated implantation failure, $16.2 \%$ vs. $10.3 \%$ for recurrent miscarriages, and $1.7 \%$ vs. $1.0 \%$ for previous affected pregnancies $(P=$ 0.496).

Patients from Groups 1 and 2 were comparable in terms of age $(38.7 \pm 3.6$ vs. $38.3 \pm 4.0)$. The mean number of metaphase II (MII) oocytes recovered
$(13.1 \pm 4.5$ vs. $13.4 \pm 5.4)$, fertilized oocytes $(9.5 \pm 3.6$ vs. $9.7 \pm 4.5)$, and developing D3 embryos $(8.3 \pm 3.4$ vs. $9.0 \pm 4.6$ ) were also similar (Table 1 ). All patients in Group 1 achieved embryo biopsy while eight patients from Group 2 (3.9\%) did not, as no embryo developed to the blastocyst stage. The indication for all these eight patients was advanced maternal age.

As shown in Table 2, a mean of $8.3 \pm 3.4$ embryos was analysed per cycle in Group 1 while $4.7 \pm 3.1$ were analysed in Group 2, this comparison being statistically different. A lower percentage of biopsied embryos were successfully diagnosed in Group 1 ( $89.4 \%$ vs. $96.1 \%)$ due to either amplification failure or low confidence profiles. The euploidy rate observed in Group 1 was lower when compared with Group 2 (20.1\% vs. $38.3 \%$ ), and complex aneuploidies (involving $\geq 3$ chromosomes) were more frequent at the cleavage stage $(50.1 \%$ vs. $14.0 \%)$. In total, 37 blastocysts $(4 \%)$ were diagnosed as euploid-aneuploid mosaic in Group 2 . The mean number of euploid embryos per cycle was the same between groups $(1.5 \pm 1.7$ vs. $1.7 \pm 1.8)$. However, given that not all cleavage-stage embryos with a euploidy diagnosis reached the blastocyst stage, fewer blastocysts were finally available per cycle (transferred and/or frozen) in Group $1(1.1 \pm 1.3 \mathrm{vs}$. $1.7 \pm 1.8$ ) (Table 2).

There were fewer cycles to transfer in Group 1 $(59.8 \%$ vs. $70.6 \%)$ and more embryos were replaced per transfer in this group $(1.4 \pm 0.5$ vs. $1.2 \pm 0.4)$. From 180 blastocysts warmed for deferred transfer in Group 2, 97.8\% survived (re-expanding or reexpanded blastocysts $2 \mathrm{~h}$ post-warming) and could be transferred. After first euploid blastocyst transfer clinical pregnancy rate per cycle was significantly lower in Group 1 (33.5\% vs. 46.1\%), but no differences in clinical pregnancy rate per transfer ( $56.1 \%$ vs. $65.3 \%)$ and implantation rate $(50.3 \%$ vs. $61.4 \%)$ were detected between groups. Multiple pregnancy ( $28.3 \%$ vs. $14.9 \%)$, miscarriage (10\% vs. $18.1 \%)$, and ectopic pregnancy rate $(1.7 \%$ vs. $3.2 \%)$ showed no significant differences. Live birth rate per cycle $(29.6 \%$ vs. $35.3 \%)$ and per transfer $(49.5 \%$ vs. $50.0 \%)$ were also similar between both groups (Table 3).

However, when taking into account successive transfers, cumulative delivery rate per initiated cycle with at least one live birth was ultimately higher in Group 2 (34.1\% vs. 44.6\%) (Table 3).

\section{Discussion}

The transition from cleavage-stage to blastocyst-stage biopsy is being embraced by many IVF laboratories, given its promising results. However, for laboratories with a well established cleavage-stage biopsy protocol and good results, this PGT-A strategy may be difficult 
Table 1 Cycles characteristics

\begin{tabular}{lccc}
\hline & Group $1(n=179)$ & Group 2 $(n=204)$ & $P$-value \\
\hline Maternal age $\left(n \pm \mathrm{SD}^{a}\right)$ & $38.7 \pm 3.6$ & $38.3 \pm 4.0$ & 0.367 \\
MII oocytes $n\left(\right.$ mean $\left.\pm \mathrm{SD}^{a}\right)$ & $2347(13.1 \pm 4.5)$ & $2731(13.4 \pm 5.4)$ & 0.881 \\
Fertilized oocytes $n\left(\right.$ mean $\left.\pm \mathrm{SD}^{a}\right)$ & $1703(9.5 \pm 3.6)$ & $1983(9.7 \pm 4.5)$ & 0.840 \\
Developing D3 embryos $n\left(\operatorname{mean} \pm \mathrm{SD}^{a}\right)$ & $1487(8.3 \pm 3.4)$ & $1831(9.0 \pm 4.6)$ & 0.107 \\
Cycles with biopsiable embryos $n(\%)$ & $179(100.0)$ & $196(96.1)$ & 0.020 \\
\hline
\end{tabular}

aSD: Standard deviation.

Table 2 PGT-A results

\begin{tabular}{|c|c|c|c|}
\hline & Group $1(n=179)$ & Group $2(n=204)$ & $P$-value \\
\hline Biopsied embryos (per cycle $n \pm \mathrm{SD}^{a}$ ) & $1487(8.3 \pm 3.4)$ & $949(4.7 \pm 3.1)$ & $<0.001$ \\
\hline Embryos successfully diagnosed $n(\%)$ & $1329\left[89.4(87.1-91.0)^{b}\right]$ & $912\left[96.1\left(94.9-97.3^{b}\right)\right]$ & - \\
\hline Euploid embryos $n(\%)$ & $267\left[20.1(17.2-23.0)^{b}\right]$ & $349\left[38.3(34.5-42.0)^{b}\right]$ & - \\
\hline Embryos with complex aneuploidies $n(\%)$ & $532\left[50.1(46.3-53.9)^{b}\right]$ & $79\left[14.0(11.1-16.9)^{b}\right]$ & - \\
\hline Mosaic euploid-aneuploid embryos $n(\%)$ & N.A. & $37(4.0)$ & - \\
\hline Euploid embryos/cycle $n \pm \mathrm{SD}^{a}$ & $1.5 \pm 1.7$ & $1.7 \pm 1.8$ & 0.178 \\
\hline Available euploid blastocysts (per cycle $n \pm \mathrm{SD}^{a}$ ) & $199(1.1 \pm 1.3)$ & $349(1.7 \pm 1.8)$ & 0.001 \\
\hline
\end{tabular}

aSD: Standard deviation.

b95\% Confidence interval.

Table 3 Clinical outcomes ${ }^{a}$

\begin{tabular}{|c|c|c|c|}
\hline & Group $1(n=179)$ & Group $2(n=204)$ & $P$-value \\
\hline Cycles to transfer ${ }^{b} n(\%)$ & $107(59.8)$ & 144 (70.6) & 0.03 \\
\hline Transferred embryos $n\left( \pm \mathrm{SD}^{c}\right)$ & $153(1.4 \pm 0.5)$ & $176(1.2 \pm 0.4)$ & $<0.001$ \\
\hline Clinical pregnancies $n \%$ per transfer, $\%$ per cycle) & $60(56.1,33.5)$ & $94(65.3,46.1)$ & $0.02,0.15$ \\
\hline Implanted embryos $n(\%)$ & $77\left[50.3(41.2-59.5)^{d}\right]$ & $108\left[61.4(53.3-69.4)^{d}\right]$ & - \\
\hline Miscarriages $n(\%)$ & $6(10.0)$ & $17(18.1)$ & 0.24 \\
\hline Ectopic pregnancies $n(\%)$ & $1(1.7)$ & $3(3.2)$ & 1 \\
\hline Multiple pregnancies $n(\%)$ & $17(28.3)$ & $14(14.9)$ & 0.06 \\
\hline Live births $n$ (\% per cycle) & $53(29.6)$ & $72(35.3)$ & 0.27 \\
\hline $\begin{array}{l}\text { Cycles with at least one live birth after successive } \\
\text { transfers } n \text { (\% per cycle) }\end{array}$ & $61(34.1)$ & $91(44.6)$ & 0.04 \\
\hline
\end{tabular}

${ }^{a}$ Clinical outcomes for first euploid embryo transfers of each cycle are presented. Only the variable 'cycles with at least one live birth after successive transfers' is related to the total number of transfers in the included cycles.

${ }^{b}$ Cycles with at least one transfer.

'SD: Standard deviation.

${ }^{d} 95 \%$ Confidence interval.

to abandon. In our centre, with good results in D+3 biopsy, the aim was to assess how the strategy change could modify our PGT-A results in order to evaluate the newly implemented approach.

As shown by our results, while all cycles in Group 1 had a biopsy performed, there were eight cycles in Group 2 (3.9\%) with no embryos developing to the blastocyst stage. The possibility of not having blastocysts available for biopsy has to be communicated to patients undergoing PGT-A with trophectoderm biopsy. As previously reported, the risk of not having blastocysts available increases with maternal age (Franasiak et al., 2014) as confirmed by our results (mean maternal age in our subgroup with no biopsy: $40.6 \pm 2.8)$.
The number of biopsied embryos in Group 1 was double the number of those in Group 2. It has been demonstrated that embryo selection takes place during development to the blastocyst stage (Alikani et al., 2000). Blastocyst formation rates reported in IVF programmes are around 50\% (Van Landuyt et al., 2005; Yang et al., 2014; Swain et al., 2016). Consequently, we can expect the number of biopsied blastocysts on $\mathrm{D}+5 / 6 / 7$ to be around half of those biopsied on $\mathrm{D}+3$.

As previously reported (Forman et al., 2012), we observed an important increase in successfully diagnosed embryos in Group 2 (89.4\% vs. 96.9\%). The biopsy of multiple cells leads to a decrease in amplification failures. As fewer embryos remain undiagnosed, the need to potentially perform an additional biopsy 
becomes residual when biopsying at the blastocyst stage, thus reducing the risks associated with the extra manipulation.

Culture to the blastocyst stage has been reported to select preferably euploid embryos (Harton et al., 2013; Adler et al., 2014). In our results, the euploidy rate is clearly lower in Group 1. Furthermore, our results regarding the incidence of complex aneuploidies show that, in most cases, highly abnormal embryos are not able to develop to the blastocyst stage. However, the selection of euploid embryos by culture to blastocyst is not complete as confirmed by the euploidy rate in Group 2 (38.3\%). Only the combination of blastocyst culture and PGT-A allows the selection of euploid blastocysts (Yang et al., 2014).

Regarding mosaicism, its incidence decreases from cleavage to blastocyst stage (Bielanska et al., 2005; Northrop et al., 2010; Fragouli et al., 2011; Capalbo et al., 2013; Mertzanidou et al., 2013), although it is still remarkable in this latter stage. Its high presence in cleavage-stage embryos and the inability to detect it by single-cell analysis can lead to misdiagnosis of aneuploidy, which has been one of the reasons that $\mathrm{D}+3$ biopsy has been abandoned in favour of blastocyst biopsy (Sermon et al., 2016).

Our results from trophectoderm biopsies analysed by aCGH showed that $4.0 \%$ of the analysed blastocysts were classified as euploid-aneuploid mosaics, in accordance with results presented by other authors using the same diagnostic technique (Greco et al., 2015). Other groups performing PGT-A with next-generation sequencing (NGS) are detecting a higher percentage of mosaic blastocysts (20\%) (Munné et al., 2016). This is because aCGH allows a reliable detection of mosaicism affecting $\geq 50 \%$ of the analysed cells (Mamas et al., 2012) while more sensitive technologies such as NGS can detect lower levels ( $\geq 20 \%$ ) (PGDIS, 2016). The application of this latter techniques could improve clinical outcomes by allowing a better selection of embryos (Maxwell et al., 2016). Although the clinical significance of mosaicism is yet to be determined (Taylor et al., 2014a), mosaic euploid-aneuploid embryos have been replaced and have generated healthy live births (Greco et al., 2015; Maxwell et al., 2016). The decision to transfer euploid-aneuploid mosaic blastocysts should be taken considering the chromosomal anomaly and the chromosomes involved and after proper genetic counselling to patients is given (Besser \& Mounts, 2017).

We observed fewer cycles to transfer in Group 1 $(59.8 \%$ vs. $70.6 \%)$. Although the number of euploid embryos was statistically similar between groups, fewer blastocysts available for transfer were finally obtained in Group 1. It has been postulated that $\mathrm{D}+3$ biopsy can have a detrimental effect on embryo development (Scott et al., 2013), a fact that could explain our results showing a significant percentage of euploid D+3 biopsied embryos (24.7\%) do not reach the blastocyst stage despite the fact that only one blastomere was removed.

According to our results, and regarding embryo transfer, lower numbers of embryos were replaced per transfer in Group $2(1.4 \pm 0.5$ vs. $1.2 \pm 0.4)$, and while in this group the percentage of double embryo transfers (DET) was $22.2 \%$, in Group 1 it was $43.0 \%$ (data not shown). As a result, multiple pregnancy rate was higher, although not statistically significant, in Group 1 (28.3\% vs. $14.9 \%)$. When transferring two euploid embryos and achieving pregnancy, multiple pregnancies were very high $(58.6 \%$ in Group 1 and $66.6 \%$ in Group 2; data not shown). This fact makes evident that single euploid blastocyst transfer must be strongly recommended in PGT-A cycles.

The increase in cycles to transfer in Group 2 led to a significantly higher clinical pregnancy rate per cycle in this group. However, our results showed a similar live birth rate per cycle in both groups $(29.6 \%$ vs. $35.3 \%$ ). This can be partially explained by the fact that miscarriage rate is higher (although not statistically significant) in Group 2 for this series of results.

The fact that more embryos are available for transfer in Group 2 is a very important argument for blastocyst biopsy, as it could lead to improved cumulative outcomes. In fact, we observed a significantly higher cumulative delivery rate per initiated cycle with at least one live birth in Group 2 (34.1\% vs. 44.6\%).

In terms of cost efficiency, the trophectoderm biopsy approach would be preferable as D+3 embryos with inability to develop to the blastocyst stage will not be unnecessarily biopsied and analysed. Moreover, the fact that a deferred transfer allows the processing of multiple cases in batch, optimizing laboratory organization (time) and resources (consumables) needs also careful consideration when assessing the cost efficiency of both strategies.

One of the concerns of embracing a deferred transfer strategy is the embryo loss due to the vitrification procedure. However, with an optimal vitrification programme, our embryo loss is minimal (2.2\%), which is similar to previous reports (Cobo et al., 2012; Taylor et al., 2014b). Conversely, deferred transfer has been demonstrated to be a good strategy offering beneficial results in certain groups of patients, even when PGT-A is not performed (Roque et al., 2013). Even though this schedule may be negatively perceived by the patients, especially those coming from abroad, deferred transfer could be beneficial to them (Evans et al., 2014; Coates et al., 2017).

A PGT-A strategy with trophectoderm biopsy and deferred transfer strategy should not be implemented without taking into consideration that its success will strongly depend on having an adequate programme 
for embryo culture to the blastocyst stage, well trained embryologists for trophectoderm biopsy, an optimal cryopreservation programme, and a well organized team. Even with all these in place, the effect of a learning curve will not allow the immediate achievement of the best results.

\section{Conclusion}

As our data show, both cleavage-stage and blastocyststage biopsy approaches can offer good results. However, trophectoderm biopsy offers a more robust diagnosis and the intervention seems to cause less harm to the embryos. Moreover, more euploid blastocysts are available, which can ultimately lead to improved cumulative live birth rates.

This paper describes in detail the comparison of two PGT-A strategies: D+3 biopsy with fresh embryo transfer and trophectoderm biopsy with deferred embryo transfer. PGT-A programmes must evaluate these two strategies under their own laboratory and clinical conditions and opt for the one that is more beneficial for their patients.

\section{Acknowledgements}

This work was performed under the auspices of the Càtedra d'Investigació en Obstetrícia i Ginecologia of the Department of Obstetrics and Gynaecology, Hospital Universitari Dexeus, Universitat Autònoma de Barcelona.

\section{Financial support}

This research received no specific grant from any funding agency, commercial or not-for-profit sectors.

\section{Statement of interest}

None.

\section{References}

Adler, A., Lee, H.-L., McCulloh, D.H., Ampeloquio, E., Clarke-Williams, M., Wertz, B.H. \& Grifo, J. (2014). Blastocyst culture selects for euploid embryos: comparison of blastomere and trophectoderm biopsies. Reprod. Biomed. Online 28, 485-91.

Alikani, M., Calderon, G., Tomkin, G., Garrisi, J., Kokot, M. \& Cohen, J. (2000). Cleavage anomalies in early human embryos and survival after prolonged culture in vitro. Hum. Reprod. 15, 2634-43.
Barri, P.N., Martinez, F., Coroleu, B. \& Tur, R. (2002). The role of $\mathrm{GnRH}$ antagonists in assisted reproduction. Reprod. Biomed. Online 5(Suppl. 1), 14-9.

Besser, A.G. \& Mounts, E.L. (2017). Counselling considerations for chromosomal mosaicism detected by preimplantation genetic screening. Reprod. Biomed. Online 34, 369-74.

Bielanska, M., Jin, S., Bernier, M., Tan, S.L. \& Ao, A. (2005). Diploid-aneuploid mosaicism in human embryos cultured to the blastocyst stage. Fertil. Steril. 84, 336-42.

Boada, M., Carrera, M., De La Iglesia, C., Sandalinas, M., Barri, P.N. \& Veiga, A. (1998). Successful use of a laser for human embryo biopsy in preimplantation genetic diagnosis: report of two cases. J. Assist. Reprod. Genet. 15, 302-7.

Capalbo, A., Wright, G., Elliott, T., Ubaldi, F.M., Rienzi, L. \& Nagy, Z.P. (2013). FISH reanalysis of inner cell mass and trophectoderm samples of previously array-CGH screened blastocysts shows high accuracy of diagnosis and no major diagnostic impact of mosaicism at the blastocyst stage. Hum. Reprod. 28, 2298-307.

Capalbo, A., Romanelli, V., Cimadomo, D., Girardi, L., Stoppa, M., Dovere, L., Dell'Edera, D., Ubaldi, F.M. \& Rienzi, L. (2016). Implementing PGD/PGD-A in IVF clinics: considerations for the best laboratory approach and management. J. Assist. Reprod. Genet. 33, 1279-86.

Coates, A., Kung, A., Mounts, E., Hesla, J., Bankowski, B., Barbieri, E., Ata, B., Cohen, J. \& Munné, S. (2017). Optimal euploid embryo transfer strategy, fresh versus frozen, after preimplantation genetic screening with next generation sequencing: a randomized controlled trial. Fertil. Steril. 107, 723-30.e3.

Cobo, A., de los Santos, M.J., Castellò, D., Gámiz, P., Campos, P. \& Remohí, J. (2012). Outcomes of vitrified early cleavagestage and blastocyst-stage embryos in a cryopreservation program: evaluation of 3,150 warming cycles. Fertil. Steril. 98, 1138-46.e1.

Coroleu, B., Barri, P.N., Carreras, O., Belil, I., Buxaderas, R., Veiga, A., \& Balasch, J. (2006). Effect of using an echogenic catheter for ultrasound-guided embryo transfer in an IVF programme: a prospective, randomized, controlled study. Hum. Reprod. 21, 1809-15.

Dahdouh, E.M., Balayla, J. \& García-Velasco, J.A. (2015). Impact of blastocyst biopsy and comprehensive chromosome screening technology on preimplantation genetic screening: a systematic review of randomized controlled trials. Reprod. Biomed. Online 30, 281-9.

de Boer, K.A., Catt, JW., Jansen, RPS., Leigh, D. \& McArthur, S. (2004). Moving to blastocyst biopsy for preimplantation genetic diagnosis and single embryo transfer at Sydney IVF. Fertil. Steril. 82, 295-8.

Dokras, A., Sargent, I.L., Ross, C., Gardner, R.L. \& Barlow, D.H. (1990). Trophectoderm biopsy in human blastocysts. Hum. Reprod. 5, 821-5.

Evans, J., Hannan, N.J., Edgell, T.A., Vollenhoven, B.J., Lutjen, P.J., Osianlis, T., Salamonsen, L.A. \& Rombauts, L.J. (2014). Fresh versus frozen embryo transfer: backing clinical decisions with scientific and clinical evidence. Hum. Reprod. Update 20, 808-21. 
Forman, E.J., Hong, K.H., Treff, N.R. \& Scott, R.T. (2012). Comprehensive chromosome screening and embryo selection: moving toward single euploid blastocyst transfer. Semin. Reprod. Med. 30, 236-42.

Fragouli, E., Alfarawati, S., Daphnis, D.D., Goodall, N.-N., Mania, A., Griffiths, T., Gordon, A. \& Wells, D. (2011). Cytogenetic analysis of human blastocysts with the use of FISH, CGH and aCGH: scientific data and technical evaluation. Hum. Reprod. 26, 480-90.

Franasiak, J.M., Forman, E.J., Hong, K.H., Werner, M.D., Upham, K.M., Treff, N.R. \& Scott, R.T. Jr. (2014). The nature of aneuploidy with increasing age of the female partner: a review of 15,169 consecutive trophectoderm biopsies evaluated with comprehensive chromosomal screening. Fertil. Steril. 101, 656-63.e1.

Greco, E., Minasi, M.G. \& Fiorentino, F. (2015). Healthy babies after intrauterine transfer of mosaic aneuploid blastocysts. New Eng. J. Med. 373, 2089-90.

Handyside, A.H. (2013). 24-chromosome copy number analysis: a comparison of available technologies. Fertil. Steril. 100, 595-602.

Harton, G.L., Munné, S., Surrey, M., Grifo, J., Kaplan, B., McCulloh, D.H., Griffin, D.K., Wells, D. \& PGD Practitioners Group. (2013). Diminished effect of maternal age on implantation after preimplantation genetic diagnosis with array comparative genomic hybridization. Fertil. Steril. 100, 1695-703.

Kuwayama, M., Vajta, G., Kato, O. \& Leibo, S.P. (2005). Highly efficient vitrification method for cryopreservation of human oocytes. Reprod. Biomed. Online 11, 300-8.

Mamas, T., Gordon, A., Brown, A., Harper, J. \& SenGupta, S. (2012). Detection of aneuploidy by array comparative genomic hybridization using cell lines to mimic a mosaic trophectoderm biopsy. Fertil. Steril. 97, 943-7.

Mastenbroek, S., Twisk, M., van Echten-Arends, J., SikkemaRaddatz, B., Korevaar, J.C., Verhoeve, H.R., Vogel, N.E., Arts, E.G., de Vries, J.W., Bossuyt, P.M., Buys, C.H., Heineman, M.J., Repping, S. \& van der Veen, F. (2007). In vitro fertilization with preimplantation genetic screening. New Engl. J. Med. 357, 9-17.

Mastenbroek, S., Twisk, M., van der Veen, F. \& Repping, S. (2011). Preimplantation genetic screening: a systematic review and meta-analysis of RCTs. Hum. Reprod. Update 17, 454-66.

Maxwell, S.M., Colls, P., Hodes-Wertz, B., McCulloh, D.H., McCaffrey, C., Wells, D., Munné, S. \& Grifo, J.A. (2016). Why do euploid embryos miscarry? A case-control study comparing the rate of aneuploidy within presumed euploid embryos that resulted in miscarriage or live birth using next-generation sequencing. Fertil. Steril. 106, 14149.e5.

Mertzanidou, A., Wilton, L., Cheng, J., Spits, C., Vanneste, E., Moreau, Y., Vermeesch, J.R., \& Sermon, K. (2013). Microarray analysis reveals abnormal chromosomal complements in over $70 \%$ of 14 normally developing human embryos. Hum. Reprod. 28, 256-64.

Munné, S., Lee, A., Rosenwaks, Z., Grifo, J. \& Cohen, J. (1993). Diagnosis of major chromosome aneuploidies in human preimplantation embryos. Hum. Reprod. 8, 2185-91.
Munné, S., Grifo, J. \& Wells, D. (2016). Mosaicism: "survival of the fittest" versus "no embryo left behind". Fertil. Steril. 105, 1146-9.

Northrop, L.E., Treff, N.R., Levy, B. \& Scott, R.T. (2010). SNP microarray-based 24 chromosome aneuploidy screening demonstrates that cleavage-stage FISH poorly predicts aneuploidy in embryos that develop to morphologically normal blastocysts. Mol. Hum. Reprod. 16, 590600.

Parriego, M., Solé, M., Aurell, R., Barri, PN. \& Veiga, A. (2007). Birth after transfer of frozen-thawed vitrified biopsied blastocysts. J. Assist. Reprod. Genet. 24, 147-9.

PGDIS (2016). Preimplantation Genetic Diagnosis International Society (PGDIS) position statement on chromosome mosaicism and preimplantation aneuploidy testing at the blastocyst stage. 2016. Available at: http://www.pgdis. org/docs/newsletter_071816.html Accessed 15 July 2017.

Roque, M., Lattes, K., Serra, S., Solà, I., Geber, S., Carreras, R. \& Checa, M.A. (2013). Fresh embryo transfer versus frozen embryo transfer in in vitro fertilization cycles: a systematic review and meta-analysis. Fertil. Steril. 99, 156-62.

Scott, R.T., Upham, K.M., Forman, E.J., Zhao, T. \& Treff, N.R. (2013). Cleavage-stage biopsy significantly impairs human embryonic implantation potential while blastocyst biopsy does not: a randomized and paired clinical trial. Fertil. Steril. 100, 624-30.

Sermon, K., Capalbo, A., Cohen, J., Coonen, E., De Rycke, M., De Vos, A., Delhanty, J., Fiorentino, F., Gleicher, N., Griesinger, G., Grifo, J., Handyside, A., Harper, J., Kokkali, G., Mastenbroek, S., Meldrum, D., Meseguer, M., Montag, M., Munné, S., Rienzi, L., Rubio, C., Scott, K., Scott, R., Simon, C., Swain, J., Treff, N., Ubaldi, F., Vassena, R., Vermeesch, J.R., Verpoest, W., Wells, D. \& Geraedts, J. (2016). The why, the how and the when of PGS 2.0: current practices and expert opinions of fertility specialists, molecular biologists, and embryologists. Mol. Hum. Reprod. 22, 845-57.

Swain, J.E., Carrell, D., Cobo, A., Meseguer, M., Rubio, C. \& Smith, G.D. (2016). Optimizing the culture environment and embryo manipulation to help maintain embryo developmental potential. Fertil. Steril. 105, 571-87.

Taylor, T.H., Gitlin, S.A., Patrick, J.L., Crain, J.L., Wilson, J.M. \& Griffin, D.K. (2014a). The origin, mechanisms, incidence and clinical consequences of chromosomal mosaicism in humans. Hum. Reprod. Update 20, 571-81.

Taylor, T.H., Patrick, J.L., Gitlin, S.A., Wilson, J.M., Crain, J.L. \& Griffin, D.K. (2014b). Outcomes of blastocysts biopsied and vitrified once versus those cryopreserved twice for euploid blastocyst transfer. Reprod. Biomed. Online 29, 5964.

Treff, N.R. \& Scott, R.T. (2012). Methods for comprehensive chromosome screening of oocytes and embryos: capabilities, limitations, and evidence of validity. J. Assist. Reprod. Genet. 29, 381-90.

Van Landuyt, L., De Vos, A., Joris, H., Verheyen, G., Devroey, P. \& Van Steirteghem, A. (2005). Blastocyst formation in in vitro fertilization versus intracytoplasmic sperm injection cycles: influence of the fertilization procedure. Fertil. Steril. 83, 1397-403.

Veiga, A., Sandalinas, M., Benkhalifa, M., Boada, M., Carrera, M., Santaló, J., Barri, P.N. \& Ménézo, Y. (1997). Laser 
blastocyst biopsy for preimplantation diagnosis in the human. Zygote 5, 351-4.

Wells, D., Alfarawati, S., Fragouli, E. (2008). Use of comprehensive chromosomal screening for embryo assessment: microarrays and CGH. Mol. Hum. Reprod. 14, 703-10.
Yang, Z., Zhang, J., Salem, S.A., Liu, X., Kuang, Y., Salem, R.D. \& Liu, J. (2014). Selection of competent blastocysts for transfer by combining time-lapse monitoring and array $\mathrm{CGH}$ testing for patients undergoing preimplantation genetic screening: a prospective study with sibling oocytes. BMC Med. Genomics 7, 38. 